\title{
The ANTARES Instrument Control System for Neutron Imaging with NICOS/TANGO/LiMA Converted to a Mobile System used at Idaho National Laboratory
}

\author{
Burkhard Schillinger $^{1, a^{*}}$, Aaron Craft ${ }^{2, b}$, Jens Krüger ${ }^{1, c}$ \\ ${ }^{1}$ Heinz Maier-Leibnitz Zentrum and Physics E21, Technische Universität München, \\ Lichtenbergstr.1, 85748 Garching, Germany \\ ${ }^{2}$ Advanced Characterization and Post-Irradiation Examination Department \\ Idaho National Laboratory, USA \\ aBBurkhard.Schillinger@frm2.tum.de , ${ }^{\mathrm{b}}$ Aaron Craft@inl.gov, ${ }^{\mathrm{c}} \mathrm{Jen} s . K r u e g e r @ f r m 2 . t u m . d e$
}

Keywords: Neutron Imaging, Neutron Computed Tomography, Software, NICOS, TANGO, Entangle, LiMA, Python

Abstract. The Neutron Radiography Reactor (NRAD) at Idaho National Laboratory (INL) was designed for thermal and epithermal neutron radiography for examination of highly-radioactive irradiated nuclear fuel elements, exclusively using transfer foils with film or imaging plates. Use of digital detectors was not foreseen in the high radiation environment. Recent collaborative efforts are seeking to introduce digital neutron imaging systems and neutron computed tomography (CT). Two initial camera detector systems were built using a low-price, but highquality scientific CMOS camera with massive shielding. With no existing electronic infrastructure, it was a challenge to build standalone systems. In first tests in 2017, a Raspberry Pi computer was used as a stepper motor and CT controller inside the East Radiography Station, which crashed in the high radiation field after a few dozen of images even behind $5 \mathrm{~cm}$ of lead shielding. In 2018, the network-based and decentralized instrument control system of the ANTARES imaging facility at Heinz Maier-Leibnitz Zentrum (MLZ) of Technische Universität München was scaled down to one laptop and three Raspberry Pi computers mounted outside the radiography bay, which was sufficient to control rotation and translation stages and to control the camera and record tomography measurements. The system is based on NICOS, TANGO, entangle, and LiMA, all four free toolkits for building distributed control systems. Details of the setup are described here. The downscaled system can be used standalone at any facility.

\section{Introduction}

The 250 kW Neutron Radiography Reactor (NRAD) at Idaho National Laboratory (INL) was designed for thermal and epithermal neutron radiography for examination of highly-radioactive irradiated nuclear fuel elements [1]. Samples are remotely lowered into the radiography stations from shielding flasks or a large hot cell above by elevators, and a rail transfer system remotely positions radiography cassettes into the detector position for indirect radiography. Opening NRAD's two radiography stations, the East and North Radiography Stations (ERS and NRS, respectively), on a regular basis for user operations was not foreseen when the facility was originally designed, but recent collaborative efforts seeking to introduce digital neutron imaging systems have demonstrated the need for simplified access to the radiography stations, which is an extraordinary challenge due to the high radiation environment and associated shielding requirements, and remote control of various imaging hardware inside the radiography stations. For the first working tomography setup, it was a challenge to build a local standalone instrument control system without any existing electronic infrastructure. 


\section{The first minimalist test system}

The first system tested in 2017 used cheap, but high quality scientific CMOS camera type 'ASI178 mm cool' [2] with 3096×2080 pixels,14 bit ADC, and two-stage thermoelectric cooler in combination with a $\mathrm{ZnS}+{ }^{6} \mathrm{LiF}$ neutron scintillation screen and mirror in a box. This first experiment was meant to test whether the camera would survive in the high radiation field so a minimalist quick-and-dirty solution was employed to record tomography data: A Raspberry Pi computer was used to control a stepper motor on a rotation stage and generate a sequence of images on consecutive angular positions. Since the camera does not have a hardware trigger input, and no dedicated software was available, the free astronomy software SharpCap [3] was used to control the camera on a Windows PC and record a sequence of images. To trigger the camera, the Raspberry Pi computer was wired to the left mouse button of the Windows PC mouse and generated mouse clicks to start new images in SharpCap on the Windows PC. The Raspberry Pi had to be mounted inside the radiography bay because of cable lengths. The gamma dose rate in the neutron beam at the imaging system was $\sim 2 \mathrm{~Sv} / \mathrm{hr}$ as measured with an ion chamber, and outside the beam in the ERS also has high gamma dose rate and an ambient neutron field of $10^{4}-10^{5} \mathrm{n} / \mathrm{cm}^{2} \mathrm{~s}$ because of incoherent neutron scattering of the beam in the air. After only 44 images, it crashed in the high radiation field of the unfiltered radial beam of the ERS even behind a $5 \mathrm{~cm}$ lead shielding.

\section{The second system}

The second detector was built into a larger box with a two-mirror configuration to allow for massive shielding and also remote focusing with a translation stage carrying the camera. For the first working tomography setup, it was a challenge to build a local standalone instrument control system without any existing network infrastructure. For this, the originally distributed and network-oriented instrument control system of the ANTARES imaging facility at Heinz MaierLeibnitz Zentrum of Technische Universität München was scaled down to one laptop and three Raspberry Pi computers mounted outside the radiography bay, which was sufficient to control rotation and translation stages and to control the camera and perform image acquisition for tomography. Fig. 1a shows the double-mirror camera box mounted in the NRS and Fig. 1b shows the provisional workspace outside the bay with laptop and the Raspberry Pis and controller under the table. The laptop on the right shows a live view of the last radiography image.
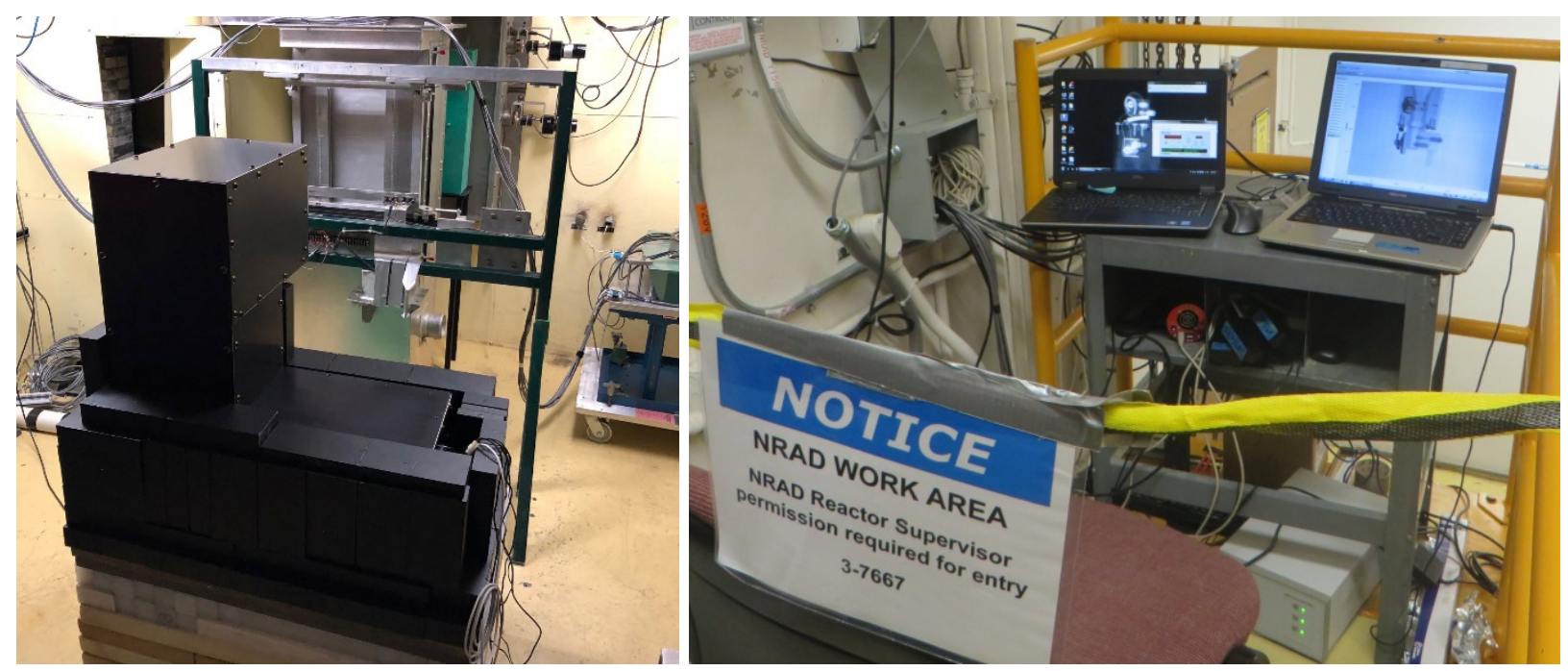

Fig.1a,b: The camera box mounted in the NRS (left) and the provisional work space with laptop and the Raspberry Pis and controller under the table (right). 


\section{The ANTARES control system reduced}

After the first generation of instruments at the Heinz Maier-Leibnitz Zentrum (MLZ) and FRM II reactor of Technische Universität München was controlled by individually tinkered solutions both in hard- and software, all instrument controls are now being standardized since 2010 using a universal network-based approach.

At the MLZ the NICOS [4] system is used as the instrument control system. It offers the control of an instrument via a command line, graphical user interface, and/or for more complex command sequences Python scripts. Additional features of NICOS are command logging, definitions of data storage position, and displaying of live data (including range selection, gamma spot filtering, and false colors). Fig. 2a,b shows a NICOS graphical interface with history and device list on the left and an open widget on the right.

The access to the hardware in our NICOS setup is done via a network based middleware: TANGO [5], a client-server framework software for instrument control, originally developed at ESRF Grenoble. The TANGO approach allows the definition of software devices which are registered by a unique name in a database of a naming service. Each device is a collection of commands and read/writable attributes, which allows to control the hardware. The translation from the logical layer to real hardware commands is done in servers, running on different machines inside the instrument network. Via the name each device may be accessed and controlled from different clients. One of the clients is the NICOS system.

In our case the LiMA [6] TANGO server is used to control the camera. LiMA is an open source framework, which supports a unified access to a large number of cameras. For all other devices like motors the entangle [7] TANGO server is used. This open source project suppports a unified access to more than 350 TANGO devices.

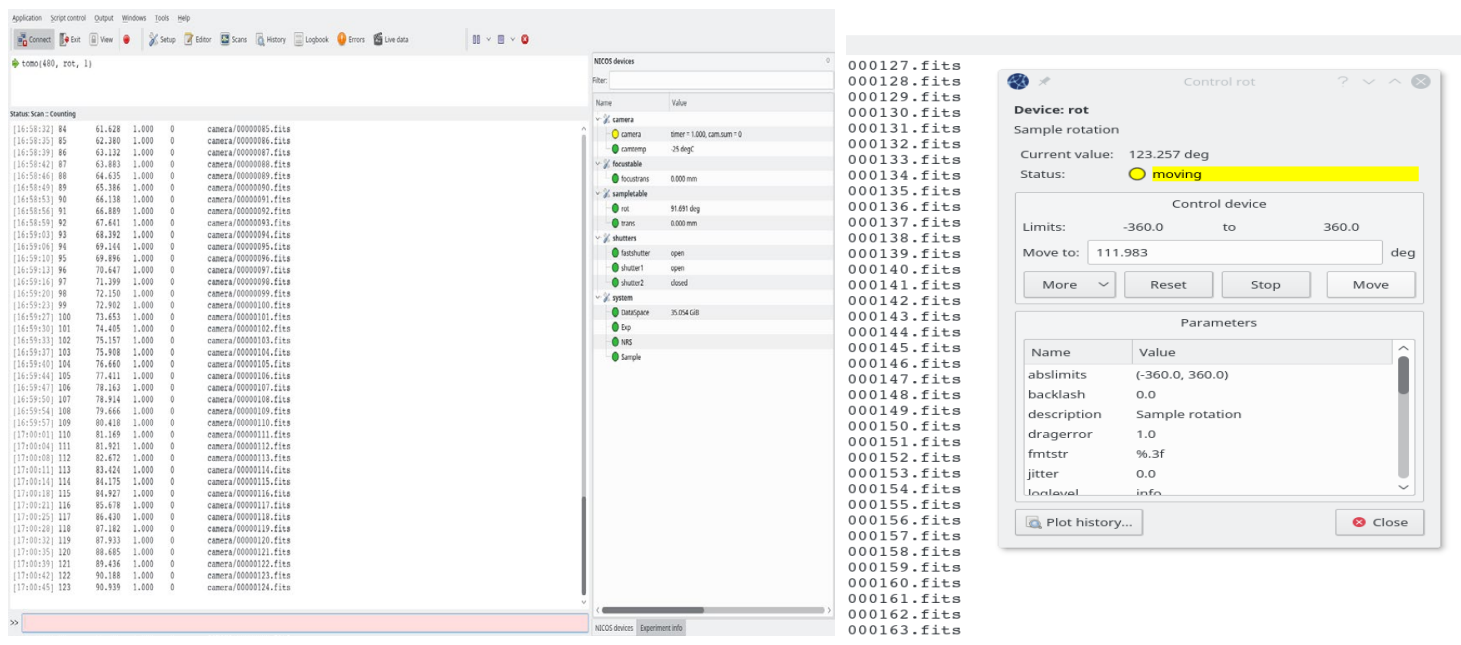

Fig.2a,b: The NICOS graphical interface with history and device list (left) and an open widget (right).

Since the INL's radiography stations were designed exclusively for remote operations outside the bay, not even basic network infrastructure existed inside beyond power outlets. For the first tomography setup, and to maintain maximum flexibility, a small network was built consisting of a network switch, three Raspberry Pi computers, and one Linux laptop (Fig.3). One Raspberry Pi acted as network controller and DNS server, one Raspberry Pi was set up as a motion controller interfacing via USB with a Thorlabs linear stage with its own controller, plus a Thorlabs multiaxis controller for the rotation table. The third Raspberry Pi was used to control the CMOS camera for radiography using the LiMA camera control software [6]. The laptop runs NICOS together with the graphical interface and was used for instrument control and data storage. 


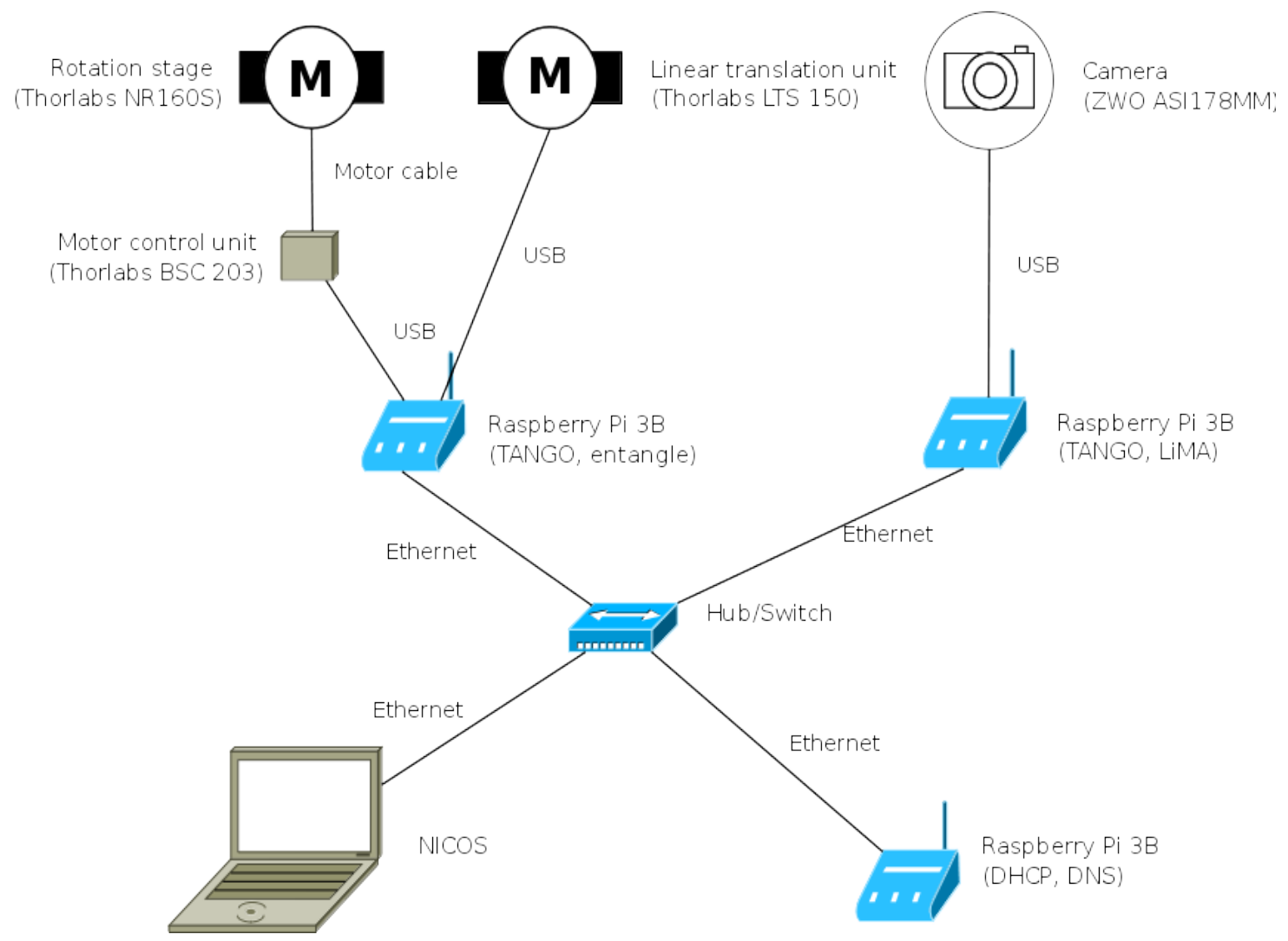

Fig.3: Components and local network of the tomography setup.

\section{First results}

The first complete 3D CT recorded at INL shows a microwave horn. Due to impending reactor shutdown, only a reduced data set of 421 images at 30 seconds each was recorded at collimation $\mathrm{L} / \mathrm{D}=185$. Fig. 4a-c shows a photo, a radiograph, and a segmented 3D view of the object.
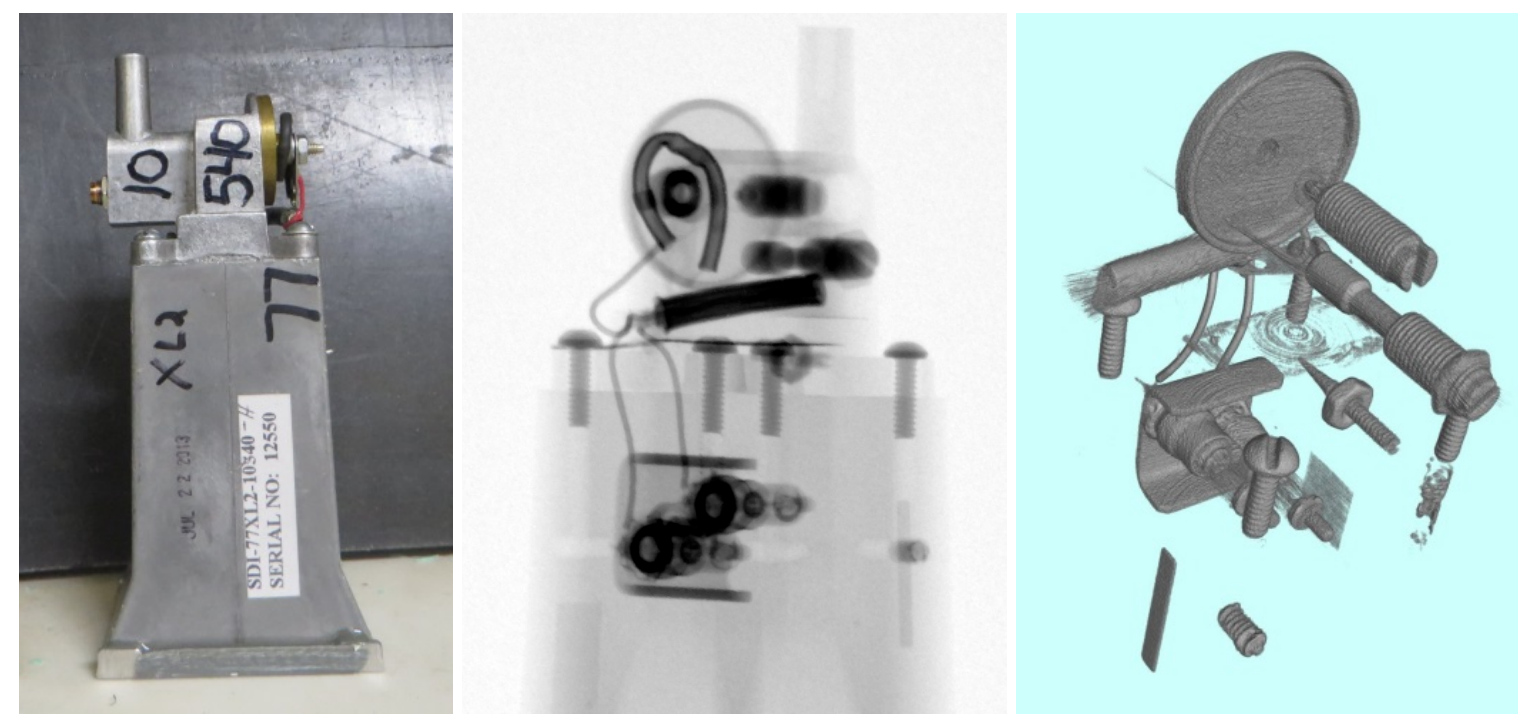

Fig.4a-c: First digital neutron CT at INL: A photo, a radiograph, and a segmented 3D view. 


\section{Conclusions and Outlook}

Using the traditional transfer method, NRAD staff could produce only 14 film radiographs per day, so collecting 421 radiographs in four hours represents a substantial improvement in the production rate by two orders of magnitude. This improvement is directly the result of the digital imaging and control systems.

Following the original TACO/TANGO philosophy, the individual software elements were developed as separate network devices. Since a dedicated instrument network is not yet available at INL, a tiny local network setup was realized to successfully achieve the first computed tomography. All components have now been combined on a single Linux computer for the next phase of introducing user operations, and the first test on-site will be conducted soon. Using the Tango/NICOS systems allows to use the already huge database of instrument servers developed at MLZ for all kinds of components like cameras, motor controllers, data interfaces and other hardware. Electronic shutter control will be added soon.

A smaller camera box with translation and rotation stage using this system was already designed and tested with this control software [8]. The construction will be simplified even more and will in the future be made available for the public for free, to be used as a universal standalone CT system.

\section{References}

[1] A.E. Craft, D.M. Wachs, M.A.Okuniewski, et al., Neutron radiography of irradiated nuclear fuel at Idaho National Laboratory, Physics Procedia 69 (2015) 483-490.

https://doi.org/10.1016/j.phpro.2015.07.068

[2] https://astronomy-imaging-camera.com/product/asi-178mm-cool/

[3] http://www.sharpcap.co.uk

[4] http://www. nicos-controls.org

[5] http://www. tango-controls.org

[6] http://lima.blissgarden.org/

[7] https://forge.frm2.tum.de/entangle/doc/entangle-master/

[8] B. Schillinger, J. Krüger, A quadruple multi-camera neutron computed tomography system at $\mathrm{MLZ}$, in this issue 